\title{
Multifrequency analysis of the Jovian electron-belt radiation during the Cassini flyby of Jupiter
}

\author{
D. Santos-Costa ${ }^{1}$, I. de Pater ${ }^{2}$, R. J. Sault ${ }^{3}$, M. A. Janssen ${ }^{4}$, S. M. Levin ${ }^{4}$, and S. J. Bolton ${ }^{1}$
}

\author{
${ }^{1}$ Southwest Research Institute, Space Science Department, San Antonio, Texas, USA \\ e-mail: [dsantoscosta; sbolton] @swri.edu \\ 2 Berkeley Astronomy Department, University of California, Berkeley Ca, USA \\ e-mail: imke@berkeley.edu \\ 3 The University of Melbourne, 3010 Victoria, Australia \\ e-mail: rsault@nrao.edu \\ 4 Jet Propulsion Laboratory/Caltech, Pasadena Ca, USA \\ e-mail: [michael.janssen;steven.m.levin]@jpl.nasa.gov
}

Received 27 March 2014 / Accepted 10 June 2014

\begin{abstract}
Aims. We examine Very Large Array (VLA) observations of Jupiter to present evidence of fluctuations in the emission produced by the electron belt in January 2001. Investigating the source of fluctuations will provide new opportunities to discuss the scenarios of temporal changes in Jupiter's synchrotron radiation (i.e., the electron belt) in future data analysis and modeling work.

Methods. To discuss the electron belt dynamics during the Cassini flyby of Jupiter, we compare the radio measurements from 2-3 January 2001 with VLA observations obtained on 20-21 December 1988, when viewing geometry and array configuration are comparable. All data are scaled to a standard Earth-Jupiter distance of 4.04 AU for comparison purposes. Brightness distribution maps with identical spatial resolutions and cartography of the equatorial radiation are constructed and examined at the wavelengths of $\sim 21 \mathrm{~cm}$ and $\sim 90 \mathrm{~cm}$.

Results. Rotation-averaged maps show that the emission from the equatorial zones of maximum intensity is weaker by $\sim 5-40 \%$, but the brightness distribution is spatially more extended on 2-3 January 2001, resulting in a total emission at both wavelengths stronger by $\sim 35 \%$. Between observation periods, the brightness distributions are observed to evolve differently during the planet rotation. Tomographic reconstructions of the equatorial radiation support our conclusion that the electron-belt population was differently distributed around the planet in December 1988 and January 2001.

Conclusions. Our analysis of VLA data sets suggests that the spatial distribution of the electron belt on 2-3 January 2001 is different from that usually observed. Our knowledge of solar activity at the time of the Cassini flyby of Jupiter suggests that the emission from the electron radiation belt was responding to external influences, most likely to solar wind structures rather than to solar radio flux, on a timescale of days to a couple of weeks. Combined results from a multisource data analysis - including spacecraft and radio observations - are needed to confirm this relationship.
\end{abstract}

Key words. radiation mechanisms: non-thermal - methods: data analysis

\section{Introduction}

From Earth, Jupiter presents itself as a powerful source of pulsed radiation at $\sim 10 \mathrm{~m}$ to $\sim 30 \mathrm{~km}$ wavelengths, which encompasses the Jovian KOM (kilometric), HOM (hectometric), and Io-DAM (decametric) and non-Io DAM radiation (e.g., Zarka 2000). The relatively long wavelength radiation is mainly generated by the cyclotron maser instabilities. At wavelengths of $\sim 1-300 \mathrm{~cm}$, Jupiter is also a strong source of thermal and non-thermal radiation. The non-thermal radiation is known as the Jovian decimetric radiation or DIM radiation. In the late 1950s, the origin of the DIM emission was identified as a source of magnetobremsstrahlung (or synchrotron) radiation emitted by energetic electrons trapped in the Jovian van Allen belt (see review by Berge \& Gulkis 1976). The energy of the radiation-belt electrons that contribute to the DIM emission typically ranges from hundreds of $\mathrm{keV}$ (i.e., barely relativistic electrons) to several hundred $\mathrm{MeV}$ (i.e., ultra-relativistic electrons) (e.g., de Pater 2004).

At wavelengths of $\sim 5$ centimeters to meters, Jupiter's DIM emission dominates the radio spectrum and the thermal component and other background sources can be easily removed from interferometric measurements. Although secondary sources of radiation are spotted off-equator with high-resolution brightness maps, more than half of the Jovian synchrotron emission is produced by near-equatorial electrons trapped at distances of $\sim 1.2-1.7$ planetary radii $\left(R_{\mathrm{J}}\right)$ with energies of $\sim 1-30 \mathrm{MeV}$ (e.g., de Pater 1990, 1991; Leblanc et al. 1997; Santos-Costa et al. 2009; Girard et al. 2012). At wavelengths shorter than $\sim 5 \mathrm{~cm}$, Jupiter becomes a strong source of thermal emission and groundbased telescopes have more difficulty measuring the Jovian synchrotron radiation. Nonetheless, with the help of proven methods to subtract the thermal component, Jupiter's synchrotron radiation has been successfully mapped at $\sim 1-4 \mathrm{~cm}$ since the $1990 \mathrm{~s}$ 
(e.g., de Pater et al. 2001; de Pater \& Dunn 2003; Kloosterman et al. 2008).

During the Cassini flyby of Jupiter in December 2000January 2001, Jupiter's synchrotron emission was mapped with the Cassini radar instrument (Elachi et al. 2004) at a wavelength of $2.2 \mathrm{~cm}$ (Janssen et al. 2001; Bolton et al. 2002). In conjunction with the Cassini radar observations, the Jovian electron belt emission was monitored with the Very Large Array (VLA) and the spatial distribution was mapped at $\sim 21$ and $\sim 92 \mathrm{~cm}$ (Janssen et al. 2001; Bolton et al. 2002). Near the time of the spacecraft observations, single-dish total flux density measurements were also obtained with a combination of the Goldstone Apple Valley Radio Telescope (GAVRT) antenna and the NASA Deep Space Network operating at 2.2, 3.5, and $13 \mathrm{~cm}$ wavelengths (Klein et al. 2001). A short-term dip in the total intensity at $13 \mathrm{~cm}$ was observed from $\sim$ January 20 to $\sim$ February 20, 2001 (Klein et al. 2001), but to date, no explanation has been provided for this unusual period of variability. The present paper is focused on reanalyzing VLA data sets and discusses evidence of temporal changes in the synchrotron radiation that had already occurred at the time of the observations during the first week of January 2001.

Time variations in Jupiter's synchrotron radiation have been reported on scales of days to months to years since the early 1970s (e.g., Gérard 1970; Klein et al. 1972, 1989). Temporal changes are more often witnessed with single-dish measurements. However, interferometric measurements - when combined with advanced techniques of tomographic image reconstruction - provide a valuable resource to study the longitudinal, radial, and temporal changes in the Jovian synchrotron radiation. This was the case during the collision of Comet ShoemakerLevy 9 with Jupiter in July 1994 (e.g., Sault et al. 1997a; de Pater et al. 1997a; de Pater and Brecht 2001) and the impact of an asteroid with Jupiter in July 2009 (e.g., Santos-Costa et al. $2011 \mathrm{a}, \mathrm{b})$. In the data analysis presented here, we also combine interferometric observations and a tomographic reconstruction technique to examine the dynamical behavior of the Jovian electron belt in early January 2001.

The following sections discuss observations with the VLA that show that Jupiter's synchrotron emission was different during the Cassini flyby of Jupiter. Investigating the source of fluctuations in early January 2001 will provide new opportunities to re-examine the scenarios of temporal changes in Jupiter's synchrotron radiation (e.g., Bolton et al. 1989; Santos-Costa et al. 2008; Kita et al. 2013, and references therein). The results of our data analysis will help constrain models of Jupiter's electron belt. The conclusions of the present paper are also valuable to the interpretation of any single-dish measurements obtained during the Cassini flyby of Jupiter.

\section{Observations and data processing}

We process and examine observations of Jupiter that were obtained with the Very Large Array (Table 1). The VLA consists of 27 dish-shaped antennae, arranged in a Y-shaped configuration. For the principal configurations $\mathrm{D}, \mathrm{C}, \mathrm{B}$, and $\mathrm{A}$, the maximum antenna separations are $1.0 \mathrm{~km}, 3.4 \mathrm{~km}, 11.1 \mathrm{~km}$, and $36.4 \mathrm{~km}$, respectively. The emission radiated by the electron belt is typically observed at the wavelengths of $\sim 6, \sim 20$, and $\sim 90 \mathrm{~cm}$, i.e., in $C, L$, and $P$ bands. Observation times are usually requested (but not always allocated) when the VLA is respectively in the C-, B-, and A-array configurations to obtain the best image resolution while retaining the sensitivity to the planet's large-scale structure.
The VLA observations discussed in this paper were carried out on 2-3 January 2001 simultaneously at $21.1 \mathrm{~cm}$ and $92.4 \mathrm{~cm}$ (i.e., respectively at $1.425 \mathrm{GHz}$ ( $L$ band) and $0.325 \mathrm{GHz}$ $(P$ band $))$. Jupiter was monitored during a complete planet rotation. Earth was at a Jovian declination, $D_{E}$, of $\sim 3.4^{\circ}$ and at a distance of $\sim 4.26 \mathrm{AU}$ from Jupiter. The VLA was in the A-array configuration (Table 1). For a radio source such as Jupiter, the large-scale structure is not completely captured in $L$ band because the A-array is missing the short spacings at which the entire source can be seen. Nevertheless, the A-array configuration provides fairly good image resolutions when mapping the Jovian synchrotron radiation in $P$ and $L$ bands. During the observations of January 2001, the radio sources $1331+305$ (i.e., the IAU name of the quasi-stellar object 3C 286) and 0318+164 were used as the primary and secondary calibrators, respectively.

It is well known that Jupiter's brightness distribution changes with $D_{E}$ (Dulk et al. 1999; Kloosterman et al. 2005). To investigate changes in Jupiter's synchrotron radiation that are not related to $D_{E}$, we compare the VLA observations of January 2001 with measurements carried out in December 1988 at 20.5 and $84.3 \mathrm{~cm}$ (i.e., simultaneously in $L$ and $P$ bands), when $D_{E}$ was $\sim 3.6^{\circ}$ (Table 1). Contrary to the observations in January 2001, Jupiter could not be observed over a full rotation. Instead, the planet was observed on two consecutive days to cover a full rotation. For the observations of December 1988, the radio sources $0137+331$ (the quasi-stellar object 3C 48) and $0316+161$ were used as the primary and secondary calibrators, respectively. The December 1988 and January 2001 observations currently provide the only VLA data sets available in $L$ and $P$ bands, during periods when $D_{E}$ was $\sim 3.5^{\circ}$ and the VLA was in the A-array configuration.

Using the data reduction techniques and reconstruction methods outlined in Santos-Costa et al. (2011b), we build radio maps and equatorial cartography of brightness distribution with the observations of December 1988 and January 2001. First, we download the 1988 and 2001 VLA raw data using the online National Radio Astronomy Observatory (NRAO) archive query tool. Once downloaded, the VLA export files are converted to MS (Measurement Set) files using Common Astronomy Software Applications (CASA; Ott \& Kern 2013), and Flexible Image Transport System (FITS) uv files are subsequently created. The FITS uv files are flagged and calibrated. Clean images (or radio maps) are constructed with the Multichannel Image Reconstruction Image Analysis and Display (MIRIAD) software package (Sault et al. 1995). To only map Jupiter's synchrotron radiation, limb-darkened disk models with blackbody temperatures of 525 and $350 \mathrm{~K}$ (de Pater 1990, p. 361, Fig. 4) are used to subtract the thermal emission from the $P$ - and $L$-band observations, respectively. We use symmetrical Gaussian beams to restore the clean components. The beam sizes are adjusted so that the spatial resolution, relative to Jupiter, is equal for any maps constructed in the same radio band. The resolving beam size, therefore, varies from observation period to observation period (Table 2). This approach enables us to properly discern the differences in the Jovian synchrotron when the distance separating Earth and Jupiter varies between observation periods (e.g., Kloosterman et al. 2005). We also scale all data to a standard Earth-Jupiter distance of $4.04 \mathrm{AU}$ by multiplying all intensities by $\left(\delta_{\text {ej }} / 4.04\right)^{2}$, with $\delta_{\text {ej }}$ the geocentric distance at the time of the observations (Table 1). During the data reduction, effects of the magnetic field wobble are corrected and the resultant radio maps have a vertical magnetic axis.

Radio map units for the intensity are Jy/beam (i.e., flux density per synthesized beam). For VLA observations, the total 
Table 1. Logs of selected VLA observations.

\begin{tabular}{cccccccc}
\hline \hline Year & $\begin{array}{c}\text { UT date } \\
\text { month:d/h-d/h }\end{array}$ & $\begin{array}{c}\text { VLA } \\
\text { conf. }\end{array}$ & $\begin{array}{c}\text { Radio } \\
\text { bands }\end{array}$ & $\begin{array}{c}D_{E} \\
(\text { deg. })\end{array}$ & $\begin{array}{c}\text { long. }\left({ }^{\circ}\right) \\
(\text { Syst. III) }\end{array}$ & $\begin{array}{c}\text { Disk size } \\
\text { (arcsec) }\end{array}$ & $\begin{array}{c}\text { Dist. } \\
\text { AU }\end{array}$ \\
\hline \multirow{2}{*}{1988} & Dec.:20/01:30-20/08:15 & A & $L, P$ & $\sim 3.58$ & {$[\sim 095 ; \sim 336]$} & 47.41 & 4.16 \\
& Dec.:21/02:15-21/09:10 & A & $L, P$ & $\sim 3.57$ & {$[\sim 269 ; \sim 163]$} & 47.30 & 4.17 \\
1997 & May:06/10:00-06/17:30 & B & $L, C$ & $\sim-0.06$ & {$[\sim 186 ; \sim 0.97]$} & 38.92 & 5.07 \\
& May:07/10:00-07/18:15 & B & $L, C$ & $\sim-0.05$ & {$[\sim 337 ; \sim 275]$} & 39.04 & 5.05 \\
& May:11/10:15-11/17:00 & B & $L, C$ & $\sim-0.02$ & {$[\sim 228 ; \sim 112]$} & 39.54 & 4.99 \\
& May:12/09:45-12/17:00 & B & $L, C$ & $\sim-0.01$ & {$[\sim 000 ; \sim 262]$} & 39.66 & 4.97 \\
2001 & Jan.:02/22:50-03/09:50 & A & $L, P$ & $\sim 3.44$ & $360^{\circ}$-coverage & 46.26 & 4.26 \\
\hline
\end{tabular}

Notes. Are listed: dates, array configuration, radio bands, Earth declination, longitude coverage, Jupiter's disk size, and Jupiter-Earth geocentric distance.

Table 2. Peak intensities, flux density, and restoring beams for rotation-averaged images and maps at different CMLs.

\begin{tabular}{|c|c|c|c|c|c|c|}
\hline Period & $\begin{array}{l}\text { Radio } \\
\text { band }\end{array}$ & $\begin{array}{l}\text { CML } \\
\text { (deg.) }\end{array}$ & $\begin{array}{c}\text { East } \\
\text { lobe } \\
(\mathrm{mJy} / \mathrm{beam})\end{array}$ & $\begin{array}{c}\text { West } \\
\text { lobe } \\
\text { (mJy/beam) }\end{array}$ & $\begin{array}{l}\text { Flux } \\
\text { density } \\
\text { (Jy) }\end{array}$ & $\begin{array}{c}\text { Restoring } \\
\text { beam } \\
(\operatorname{arcsec})\end{array}$ \\
\hline \multirow{10}{*}{$\begin{array}{l}1988 \text {-Dec.-20 } \\
\text { to } \\
\text { 1988-Dec.-21 }\end{array}$} & \multirow[t]{5}{*}{$\bar{P}$} & All & $70.8 \pm 5.9$ & $82.2 \pm 5.8$ & $3.61 \pm 0.26$ & 6.20 \\
\hline & & 000 & $229.0 \pm 16.2$ & $246.7 \pm 17.5$ & $4.93 \pm 0.35$ & 12.40 \\
\hline & & 120 & $199.6 \pm 14.1$ & $193.6 \pm 13.7$ & $3.74 \pm 0.26$ & 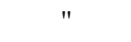 \\
\hline & & 240 & $236.8 \pm 16.8$ & $194.7 \pm 13.8$ & $4.54 \pm 0.32$ & 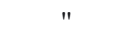 \\
\hline & & 320 & $215.0 \pm 15.2$ & $270.0 \pm 19.1$ & $4.91 \pm 0.35$ & " \\
\hline & \multirow[t]{5}{*}{$L$} & All & $30.6 \pm 2.2$ & $42.8 \pm 3.0$ & $3.79 \pm 0.27$ & 3.70 \\
\hline & & 000 & $92.3 \pm 6.5$ & $121.4 \pm 8.6$ & $2.84 \pm 0.20$ & 7.80 \\
\hline & & 120 & $100.9 \pm 7.1$ & $125.4 \pm 8.9$ & $3.13 \pm 0.22$ & " \\
\hline & & 240 & $99.5 \pm 7.0$ & $101.7 \pm 7.2$ & $2.62 \pm 0.19$ & " \\
\hline & & 320 & $101.9 \pm 7.2$ & $138.2 \pm 9.8$ & $3.31 \pm 0.23$ & $"$ \\
\hline \multirow{10}{*}{$\begin{array}{l}\text { 2001-Jan.-02 } \\
\text { to } \\
\text { 2001-Jan.-03 }\end{array}$} & \multirow[t]{5}{*}{$P$} & All & $65.2 \pm 4.6$ & $71.8 \pm 5.1$ & $5.59 \pm 0.40$ & 6.06 \\
\hline & & 000 & $239.6 \pm 16.9$ & $255.8 \pm 18.1$ & $6.79 \pm 0.48$ & 12.11 \\
\hline & & 120 & $229.3 \pm 16.2$ & $254.7 \pm 18.0$ & $6.78 \pm 0.48$ & " \\
\hline & & 240 & $159.6 \pm 11.3$ & $146.3 \pm 10.4$ & $4.92 \pm 0.35$ & . \\
\hline & & 320 & $256.7 \pm 18.2$ & $254.3 \pm 18.0$ & $6.48 \pm 0.46$ & " \\
\hline & \multirow[t]{5}{*}{$L$} & All & $29.6 \pm 2.1$ & $31.3 \pm 2.2$ & $6.02 \pm 0.43$ & 3.61 \\
\hline & & 000 & $85.8 \pm 6.1$ & $116.5 \pm 8.2$ & $3.46 \pm 0.25$ & 7.62 \\
\hline & & 120 & $101.0 \pm 7.1$ & $103.6 \pm 7.3$ & $3.32 \pm 0.23$ & $"$ \\
\hline & & 240 & $118.8 \pm 8.4$ & $103.0 \pm 7.3$ & $5.79 \pm 0.41$ & " \\
\hline & & 320 & $98.7 \pm 7.0$ & $121.5 \pm 8.6$ & $4.11 \pm 0.29$ & 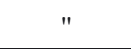 \\
\hline
\end{tabular}

error is estimated by combining in quadrature three different terms of error (e.g., Santos-Costa et al. 2011b). The systematic and absolute calibration errors $\Delta \mathrm{Err}_{\text {syst }}$ and $\Delta \mathrm{Err}_{\text {calib }}$ are usually good to a level of $5 \%$ or better in $L$ and $P$ bands (e.g., de Pater 1991). At a wavelength of $\lambda_{\mathrm{obs}}$, error due to the noise is $\Delta \operatorname{Err}_{\text {noise }}(K)=\left(\sigma_{\text {noise }} / \Omega_{\mathrm{A}}\right) \cdot\left(\left(\lambda_{\mathrm{obs}}\right)^{2} / 2 \mathrm{k}\right)$, with $k$ the Boltzmann constant and $\Omega_{\mathrm{A}}=\pi \theta_{\mathrm{Ba}} \theta_{\mathrm{Bb}} /(4 \ln (2))$ the beam solid angle of an elliptical Gaussian beam with dimensions $\theta_{\mathrm{Ba}}$ and $\theta_{\mathrm{Bb}}$ (see NRAO Green Book 1992). During the data reduction, the MIRIAD task INVERT determines the root mean square noise term $\sigma_{\text {noise }}(\mathrm{Jy} /$ beam $)$.

The reconstructed cartography depicts the equatorial brightness distribution of the volume emissivity $\varepsilon$, which has units $\mathrm{WHz}^{-1} \mathrm{~m}^{-3} \mathrm{sr}^{-1}$. A tomographic technique computes the equatorial distribution using the singular value decomposition method and the level of radiation measured on radio maps are used as inputs (Santos-Costa et al. 2011b). The tomographic images are computed with great accuracy, which means that computational errors are relatively small and errors on the emissivity are similar in magnitude to those on the intensity of the 2D maps. In
Sects. 3 and 4, any variations of $\sim 7-8 \%$ or less can be considered insignificant or within data errors.

\section{Data analysis results from rotation-averaged images}

Figure 1 presents rotation-averaged maps in total intensity of Jupiter's synchrotron emission that were constructed for the VLA observations of December 1988 and January 2001 (Table 1). All four spatial distributions present similarities that are characteristic of the DIM emission. Equatorial source of radiation and secondary emissions located at high-latitudes are typically observed on either or both sides of the planet. The main features in the brightness distribution of Jupiter's synchrotron radiation were discovered in the 1980s and 1990s, when highresolution images in total intensity or linear polarization were first obtained at 6, 13, 20, and $90 \mathrm{~cm}$ (e.g., de Pater 1990, 1991; Leblanc et al. 1997; Santos-Costa et al. 2009) and later at $2.2 \mathrm{~cm}$ 

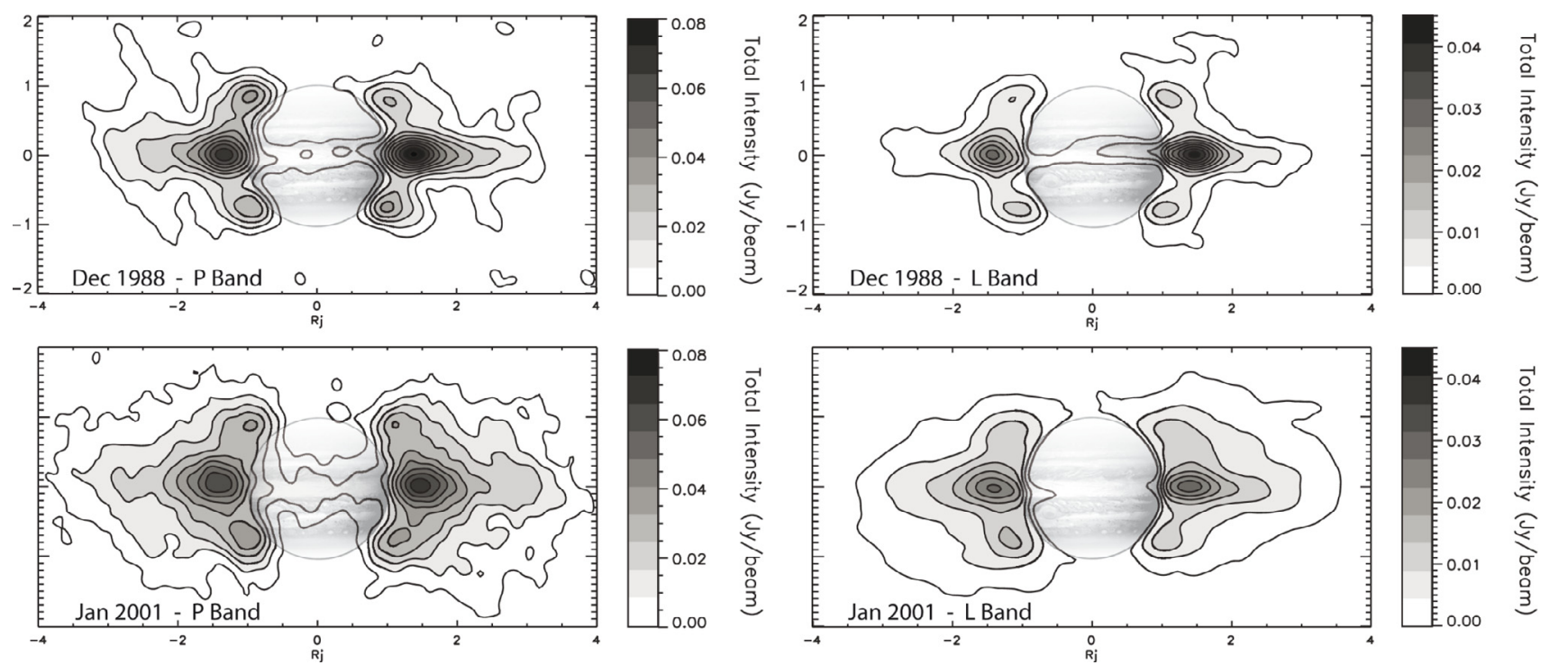

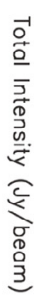

Fig. 1. Rotation-averaged images in total intensity of Jupiter's synchrotron radiation in $P$ band (left-hand panels) and $L$ band (right-hand panels) for periods when $D_{E}$ was $\sim 3.5^{\circ}$ and the VLA was in the A-array configuration. Radio maps at the same wavelength were degraded to the same image resolution. The planet disk was added to highlight the spatial distribution of the radiation around Jupiter. The main features of Jupiter's synchrotron radiation are displayed in all four radio maps: a primary source of radiation near the equator and secondary sources at high latitudes appear on either or both sides of the planet. Overall, the brightness distributions in mid-December 1988 are more east-west asymmetric and confined to the equator than they are in early January 2001.
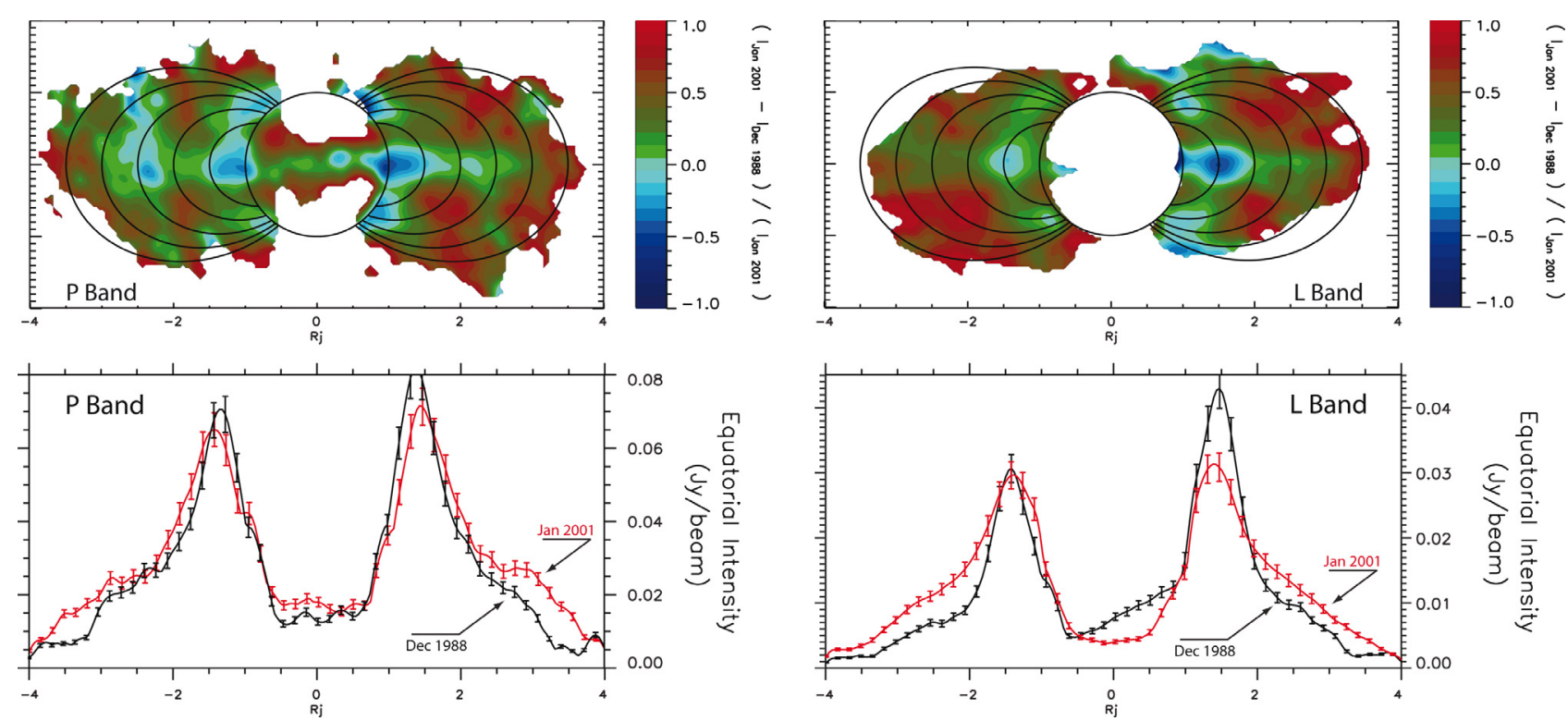

Fig. 2. Top panels: color maps of the difference in the Jovian synchrotron intensity between the 1988 and 2001 observations, normalized to the radiation measured in 2001. Overlapping are the dipole field lines crossing the equator at radial distances of $1.5,2,2.5,3$., and 3.5 $R_{\mathrm{J}}$. Bottom panels: radial profiles of the intensity measured along the equator.

(de Pater \& Dunn 2003; Kloosterman et al. 2008). For each radio map in Fig. 1, the peak intensity is located at the equator on the right-hand (i.e., west) side of the planet.

During periods when $D_{E}$ is $\sim 3.5^{\circ}$ and the VLA is in the A-array configuration, radio images of Jupiter's synchrotron emission are expected to display more similarities than we actually observe in Fig. 1. First, we notice that the equatorial peak emissions are more intense by a factor from $\sim 2$ up to $\sim 6$ on the right-hand (i.e., west) side of the planet in December 1988 (see Table 2). The radio images subsequently appear to be more eastwest asymmetric on 20-21 December 1988 than they are on 2-3 January 2001 (Fig. 1, Table 2).
We also observe that the spatial distributions are particularly more extended in latitude in January 2001 than they are in December 1988. The upper panels of Fig. 2 map the differences in intensity between the two observation periods. The differences, which are normalized to the radiation measured in 2001, are only estimated for pixels with intensity greater than the background signal. For the regions of equatorial maxima and secondary sources of emission, the radiation is weaker by up to $\sim 40 \%$ on $2-3$ January 2001 . Outside these regions, the radiation becomes more intense by up to $90 \%$. The temporal decrease and increase in intensity in different radiation zones result in a brightness distribution that is spatially more extended 
in January 2001. The extended radiation is produced by electrons bouncing along field lines crossing the equator at distances greater than $\sim 1.7 R_{\mathrm{J}}$. Radial profiles of the intensity measured along the equator illustrate our findings (Fig. 2, bottom panels). On 2-3 January 2001, the equatorial radiation in both bands is stronger at radial distances of $\sim 1.7-3.7 R_{\mathrm{J}}$ and the radial profiles present strong evidence of east-west asymmetry. In $P$ band the radiation peaks are closer to the planet in 1988 than in 2001, while the opposite is observed in $L$ band.

The total DIM emission is also found to be stronger during the observations of January 2001 (Table 2). This is the direct result of brightness distributions being spatially more expanded, as the radiation from the primary and secondary sources of emission are weaker for that peculiar period of observations. The total flux density in $P$ (respectively $L$ ) band, which is here estimated from the integration of the corresponding rotationaveraged brightness distribution map for pixel intensity above the sky background (e.g., Pelyushenko \& Stankevitch 1969), is $5.6 \pm 0.4$ (respectively $6.0 \pm 0.4$ ) Jansky. The total flux densities are found to be $\sim 36 \%$ more intense on 2-3 January 2001 than they are on 20-21 December 1988 (Table 2).

Once the viewing parameters and magnetic field morphology are ruled out, one believes that the differences in brightness distribution between observation periods are caused by changes in the energy and spatial distributions of the radiating electrons. Changes in the spatial distribution of the electron belt are supposedly driven by either internal or external parameters (e.g., Bolton et al. 1989; Brecht et al. 2001; de Pater and Brecht 2001; Horne et al. 2008; Santos-Costa et al. 2008, 2011; Kita et al. 2013, and references therein). Determining the exact cause(s) of changes in the radio maps in Fig. 1 will be the scope of a following paper. In the present paper, we pursue our analysis of the 1988 and 2001 VLA data sets. We particularly focus our discussion on demonstrating that changes in the electron and brightness distributions were occurring during the Cassini flyby of Jupiter and not at the time of observations in December 1988.

\section{Data analysis results from images at different longitudes}

We now demonstrate that the radiation-belt electrons were indeed differently distributed around the planet in mid-December 1988 and early January 2001 by mapping the Jovian synchrotron emission at different central meridian longitudes (CMLs). In the past, researchers have averaged over a CML range of either $12^{\circ}$ (e.g., de Pater et al. 1997a,b; Kloosterman et al. 2005), $20^{\circ}$ (e.g., Santos-Costa et al. 2009, 2011b), or 40 (e.g., Dulk et al. 1999; Santos-Costa et al. 2011b) per image to compare observations from different epochs. For our new series of radio maps, images are constructed every 40 degrees of CML, using data at $\pm 40^{\circ}$ of that CML, i.e., averaged over $80^{\circ}$ in CML (Figs. 3 and 4 ). This approach was used by Bolton et al. (2002) to examine the January 2001 VLA observations. We note that the longitude coverage in $L$ and $P$ bands are the same, since the data were taken simultaneously.

Figure 3 displays radio images at the wavelength of $L$ band and different CMLs. There is not much difference in the brightness distribution between the two epochs at some longitudes, whereas there is a considerable discrepancy at others. For instance, the radio maps at CMLs of 0 and 320 degrees do not present any significant differences in the equatorial brightness intensity. For both campaigns of observations, the west peak emissions are more intense - hereafter referred to by the term "brighter" - by $18 \%$ to $26 \%$ (Table 2 ). Contrary to the radio maps at CMLs of 0 and 320 degrees, peak intensities at CMLs of 120 and 240 degrees vary with time. At these longitudes, the peak emission on one side of the planet is steady while the radiation peak on the other side of the planet either increases or decreases by $16-19 \%$ with time (Table 2 ).

The observation of temporal variations in brightness peak intensity at only certain CMLs can be explained by longitudinal and radial changes in the equatorial brightness distribution (e.g., Santos-Costa et al. 2011a,b). These changes occur on timescales ranging from a few electron drifts around the planet (i.e., 1-2 days in the SIII longitude system) to several weeks. Longitudinal and radial changes between observation periods are confirmed in Sect. 5.

Radio maps in Fig. 3 reinforce the conclusions we have drawn from the analysis of rotation-averaged maps (Figs. 1 and 2). The emission from the equatorial zones of maximum intensity are weaker on 2-3 January 2001, except for the map at CML of $240^{\circ}$. The brightness distributions around Jupiter are spatially more extended, except for the map at CML of $120^{\circ}$. The total flux density calculated for each radio map in Fig. 3 confirms this last statement (Table 2).

In Fig. 3, we also notice that the secondary sources of radiation appear to be more detached from the equatorial brightness zones in December 1988 than they are in January 2001. This can be explained in different ways and for different reasons. For the observations in 1988, the high latitude emissions, which are controlled by the electron population bouncing at high-latitudes along field lines at distances of $\sim 2.0-2.4 R_{\mathrm{J}}$, are more intense and/or the equatorial radiation lobes are weaker in intensity. Regarding the observations in 2001, this can be explained by an enhancement in the radiation produced by electrons magnetically trapped at distances of $\sim 1.7-3.5 R_{\mathrm{J}}$ and perhaps a decrease in the equatorial radiation lobes. For typical radio maps of Jupiter's synchrotron emission in $L$ band, the equatorial radiation peaks are about three times more intense than the radiation peaks at high-latitudes (e.g., see radio maps published by de Pater et al. 1997b; Kloosterman et al. 2005; Santos-Costa \& Bolton 2008; Santos-Costa et al. 2009). Radio maps for the observation days in December 1988 verify the high latitude over the equatorial intensity ratio better, which lead us to the conclusion that the spatial distribution of the electron belt on 2-3 January 2001 is different from that usually observed. Differences in the radio maps in Fig. 3 are associated with changes in the brightness distribution that most likely occurred in January 2001 rather than in December 1988.

We observe comparable variations in Jupiter's synchrotron radiation at the wavelength of $P$ band (Fig. 4, Table 2). We clearly discern differences in the brightness distributions and the peak intensities had also varied between the two observation periods. The spatial distributions of the Jovian emission in $P$ band appear to be more extended on 2-3 January 2001 than on 20-21 December 1988. The total flux density calculated for each radio map in Fig. 4 is also more intense during the observations of January 2001 (Table 2).

\section{Data analysis results from tomographic reconstructions}

The comparison of radio maps between observation periods strongly suggests that the Jovian synchrotron emission, and subsequently the radiation-belt electron population, were differently distributed around the planet in mid-December 1988 and during the Cassini flyby of Jupiter (Figs. 3 and 4). Results from Sect. 4 further suggest that the changes in the spatial distributions most 

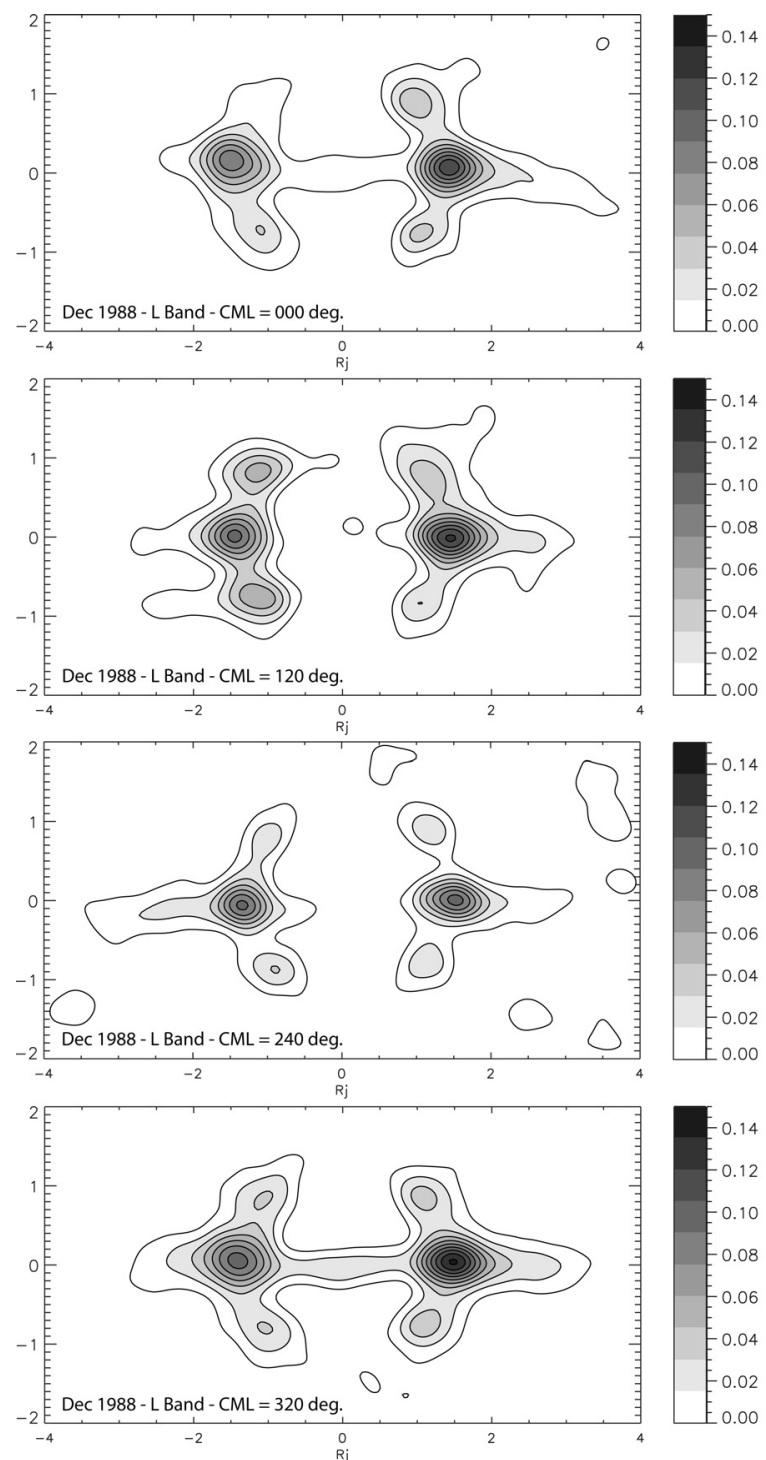
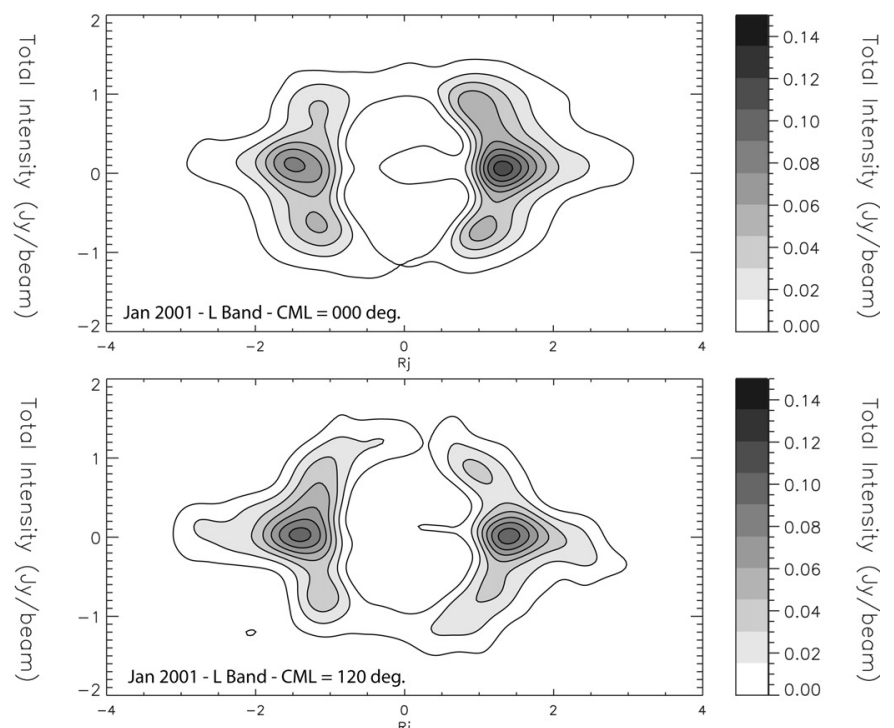

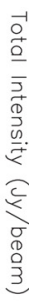
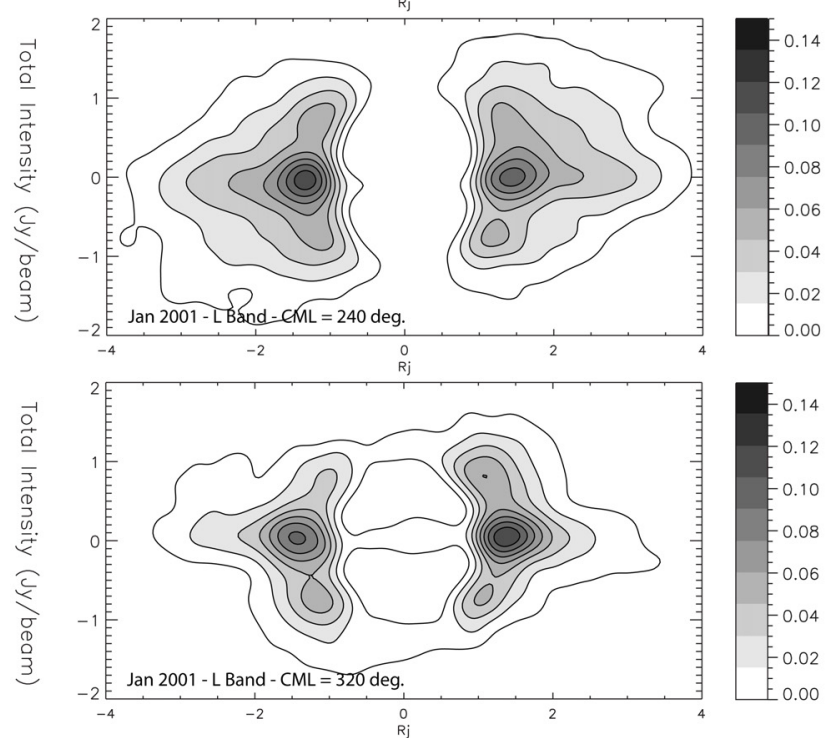

Fig. 3. Radio maps in total intensity of Jupiter's synchrotron radiation at the wavelength of $L$ band and CMLs of 0, 120, 240, and 320 degrees. For a radio map at a given CML, data at $\pm 40^{\circ}$ of that CML were used. All radio maps were degraded to the same image resolution. Differences between brightness distributions at the same CMLs are observed.

likely occurred in January 2001. To confirm these results, we examine a set of equatorial brightness distributions. The tomographic reconstruction technique used to map the equatorial radiation is described by Santos-Costa et al. (2011b). The validity of this technique is discussed in Appendix A. The VLA observations of December 1988 and January 2001 are compared to those of May 1997, which permit Jupiter's synchrotron emission to be examined in a steady state (Santos-Costa et al. 2011b) and thus will be used as our baseline observations in Sect. 5 .

Figure 5 presents the equatorial brightness distributions constructed for the VLA observations of December 1988 and January 2001. Each equatorial map was derived from a set of 18 radio maps built every 20 degrees of CML, with each radio map constructed using data at $\pm 20^{\circ}$ of that CML. For the $L$ (respectively $P$ ) band, the clean components were restored with the symmetrical Gaussian beams of the radio maps in Fig. 3 (respectively Fig. 4). The longitudinal profiles of the intensity and radial position of the brightness peak derived from Fig. 5 are compared in Fig. 6.

The cartography reconstructed for the $L$-band observations of December 1988 (Fig. 5, top left) presents more similarities with our baseline cartography (Fig. A.1, left) than does the one reconstructed for the observations of January 2001 (Fig. 5, bottom left). The top left cartography in Fig. 5 displays a main emission ring that covers the $[\sim 1.3 ; \sim 1.8] R_{\mathrm{J}}$ radial distance range and not farther out, as is observed with the cartography in Fig. A.1. The longitudinal profile of the equatorial brightness peak for the observations of 1988 (Fig. 6, top panel, dotted lines) also matches the profile reconstructed for the observations of May 1997 (Fig. 6, top panel, solid lines) better than does the one reconstructed for the observation of January 2001 (Fig. 6, top panel, long dashed lines). The dotted line reproduces the longitudinal profile depicted by the solid line within data and computational errors, except at the longitudes of $\sim 280^{\circ}-360^{\circ}$ where profiles significantly depart from each other; while the longitudinal profile of the brightness peak for the observations of 2001 (Fig. 6, top panel, long dashed lines) depart from the one for the observation period in May 1997 at all longitudes. We note, however, that the longitudinal profiles of the brightness peak's radial position for the observations of 1988 and 2001 both match the profile for the VLA observations of May 1997 in $L$ band within data and computational errors, except at the longitudes 
D. Santos-Costa et al.: Jupiter's electron-belt radiation during the Cassini flyby
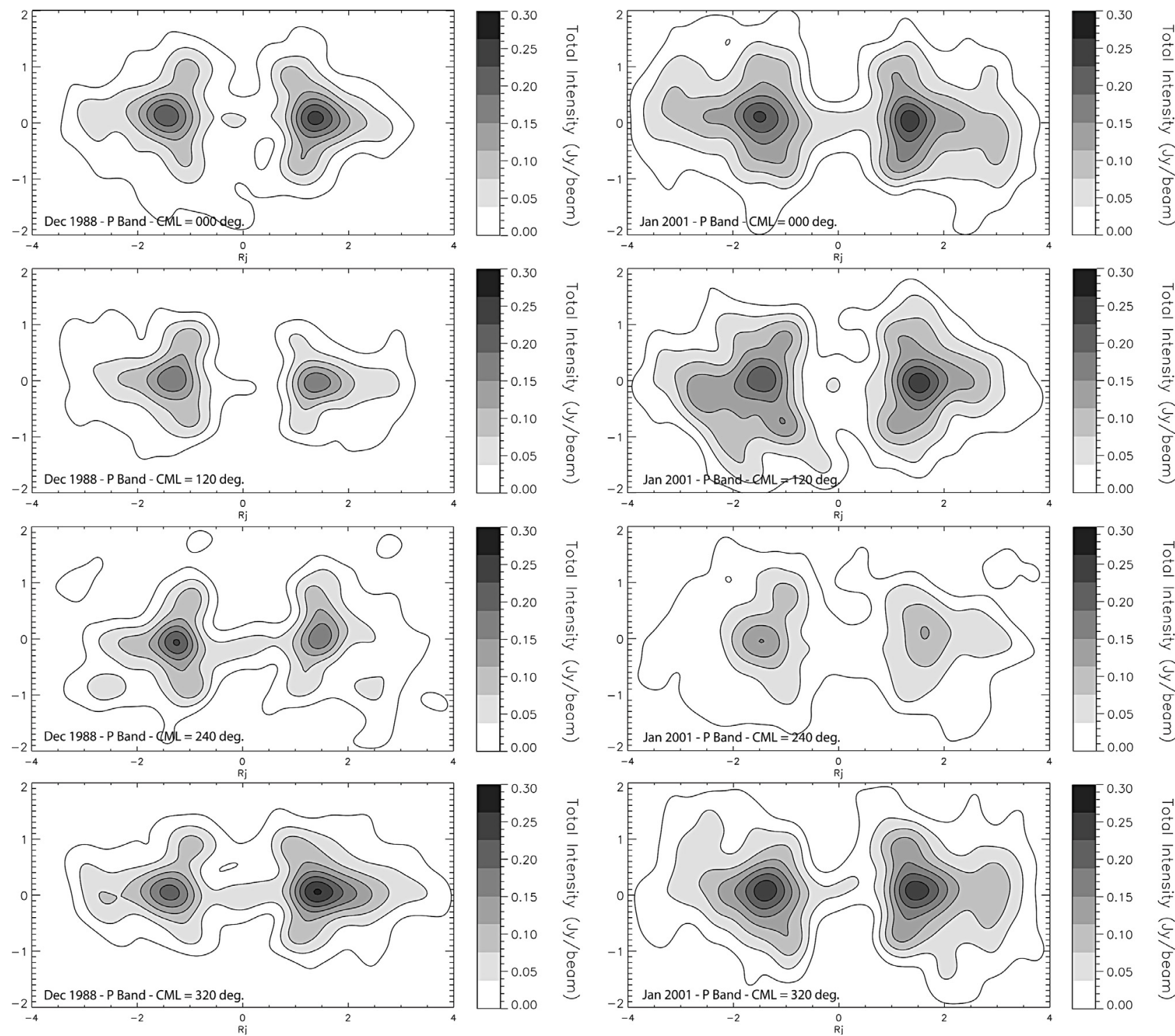

Fig. 4. Radio maps in total intensity of Jupiter's synchrotron radiation at the wavelength of $P$ band and CMLs of $0,120,240$, and 320 degrees. For a radio map at a given CML, data at $\pm 40^{\circ}$ of that CML were used. All radio maps were degraded to the same image resolution. Differences between brightness distributions at the same CMLs are observed.

of $\sim 60^{\circ}-110^{\circ}$ and $\sim 250^{\circ}-310^{\circ}$. As we have pointed out in Fig. 2 , the main emission ring at the shortest wavelength appears to be closer to the planet in January 2001 than it is in December 1988.

At the wavelength of $P$ band, we also find that the 1988 VLA observations of Jupiter's synchrotron emission present more similarities with our baseline observations than do the observations of January 2001 (Figs. 5 and 6, and Fig. A.1). Furthermore, the longitudinal profiles of the intensity and radial position in December 1988 and May 1997 (Fig. 6, bottom panel) are consistent within the error bars (we note that there are no variations with $D_{E}$; see Fig. 13 in Dunn et al. 2003).

The comparison of results of the observations of December 1988, May 1997, and January 2001 confirm that the spatial distribution of the electron belt on 2-3 January 2001 is different from that usually observed. At the shortest wavelength (i.e., $L$ band), the main difference lies outside the angular sector $\left[\sim 260^{\circ} ; \sim 340\right]$ where lower radiation levels are observed in January 2001 (Fig. 6, top panel). Furthermore, the features of the equatorial radiation for the observations of December 1988 and
May 1997 are very similar to those observed between 1981 and 1998 (e.g., de Pater \& Sault 1998; Dunn et al. 2003).

At the longest wavelength (i.e., $P$ band), the main differences between the observations of December 1988 and January 2001 are the presence of hot synchrotron spots at the SIII longitudes of $\sim 240^{\circ}-280^{\circ}$ and $\sim 310^{\circ}-340^{\circ}$ in January 2001 . The radiation level is also noticeably lower at the longitudes of $\sim 120^{\circ}-360^{\circ}$. The main emission ring is located farther away from the planet in January 2001 (Fig. 6, bottom panel). At certain longitudes, the radial displacement of the equatorial maximum reaches up to $\sim 0.2 R_{\mathrm{J}}$. This confirms the displacement of the primary zone of radiation observed in Fig. 2.

Higher radiation levels are computed at radial distances of $\sim 2-4 R_{\mathrm{J}}$ for the observations of January 2001 (bottom cartography in Fig. 5). This is the result of higher radiation being measured farther away from Jupiter (Figs. 2-4). A substantial increase in the electron belt population beyond the Jovian ring system, which would directly cause an enhancement in the synchrotron radiation in the same magnetospheric region, is a valid explanation for an extended region of radiation during the 

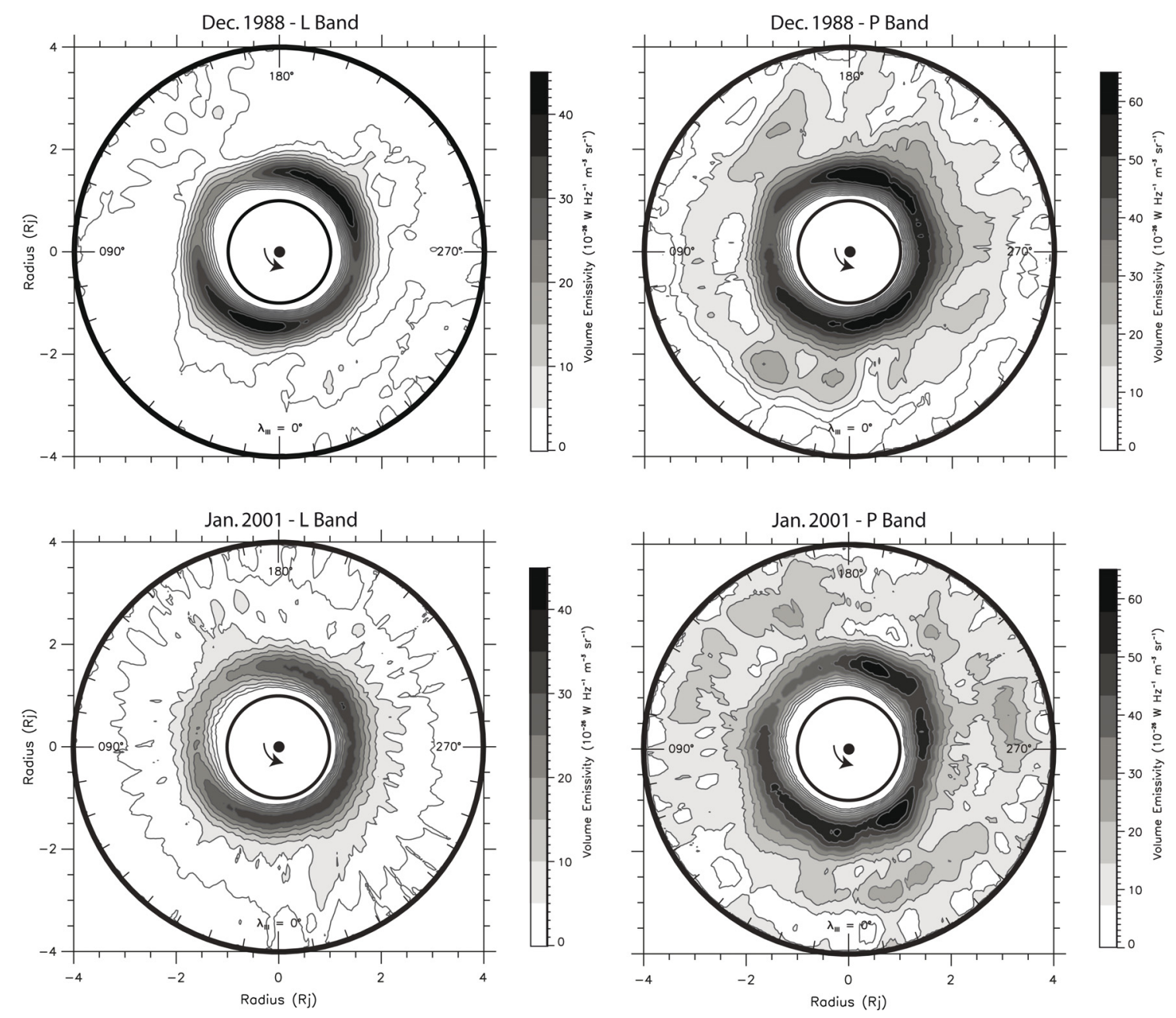

Fig. 5. Equatorial volume emissivity distributions of Jupiter's synchrotron emission at the wavelengths of $L$ band (left-hand cartography) and $P$ band (right-hand cartography) for two observation periods. The north magnetic pole is indicated by the dark dot and the planet rotation with the arrow. The coordinate system is the SIII longitude. Tomographic images show evidence of changes in the equatorial distribution between the observations carried out in December 1988 and obtained during the Cassini flyby of Jupiter in January 2001.

Cassini flyby of Jupiter. Sect. 6 presents a short discussion pointing towards what might have triggered such enhancement in Jupiter's electron-belt emission in January 2001.

\section{Discussion}

Between October 2000 and the first crossing of Jupiter's bowshock on 28 December 2000 by Cassini, interplanetary shocks and coronal mass ejections were identified in the vicinity of Jupiter with dual spacecraft observations (e.g., Krupp et al. 2004; Hanlon et al. 2004; Szegö et al. 2006). Multidata analysis results showed evidence of correlations between Jupiter's radio emissions at decametric wavelengths and auroral activity and the solar wind structures on timescales of days to a week for that period of time (e.g., Gurnett et al. 2002; Prangé et al. 2004; Pryor et al. 2005; Nichols et al. 2007).

Our analysis of the 2001 VLA data indicates that Jupiter's synchrotron emission underwent some variations during the Cassini flyby of Jupiter. The observation of changes in the emission from Jupiter's electron belt has been reported since the early 1970s (e.g., Gérard 1970; Klein et al. 1972, 1989). Correlations with solar wind on timescales of months to years (e.g., Bolton et al. 1989; Galopeau \& Gérard 2001; Santos-Costa et al. 2008) and solar radio flux on timescales of days to a week (e.g., Miyoshi et al. 1999; Tsuchiya et al. 2011; Kita et al. 2013) have been confirmed since the 1990s. However, the response of the electron belt to the solar wind on short timescales has never been considered until the present work. Such a rapid response to solar wind structures could have been mistaken as the response of the Jovian radiation belt to solar radio flux in the past, and this latter cause of variations might need to be reconsidered and ruled out in certain cases.

Fourteen days prior to the VLA observations on 2-3 January 2001, a strong interplanetary shock was spotted near Jupiter and was linked to some variability in the Jovian decametric radio signals (e.g., Gurnett et al. 2002). The Cassini spacecraft also crossed Jupiter's bow-shock four times between 28 December 2000 and 3 January 2001, which indicated rapid variations in the solar wind ram pressure during a short period of time. Our knowledge of solar wind conditions at the time of the Cassini flyby of Jupiter strongly suggests that the emission from the electron radiation belt was most likely responding to external influences on a timescale of days to a couple of weeks. The observation of shock-induced transport and energization of relativistic electrons in the magnetosphere of Earth reinforces the idea that the Jovian electron belt can similarly respond to solar wind structures (e.g., Gannon et al. 2005; Brecht et al. 2001, and references therein).

A multisource data analysis, which includes the examination of data from the Cassini and Galileo spacecraft, but also 


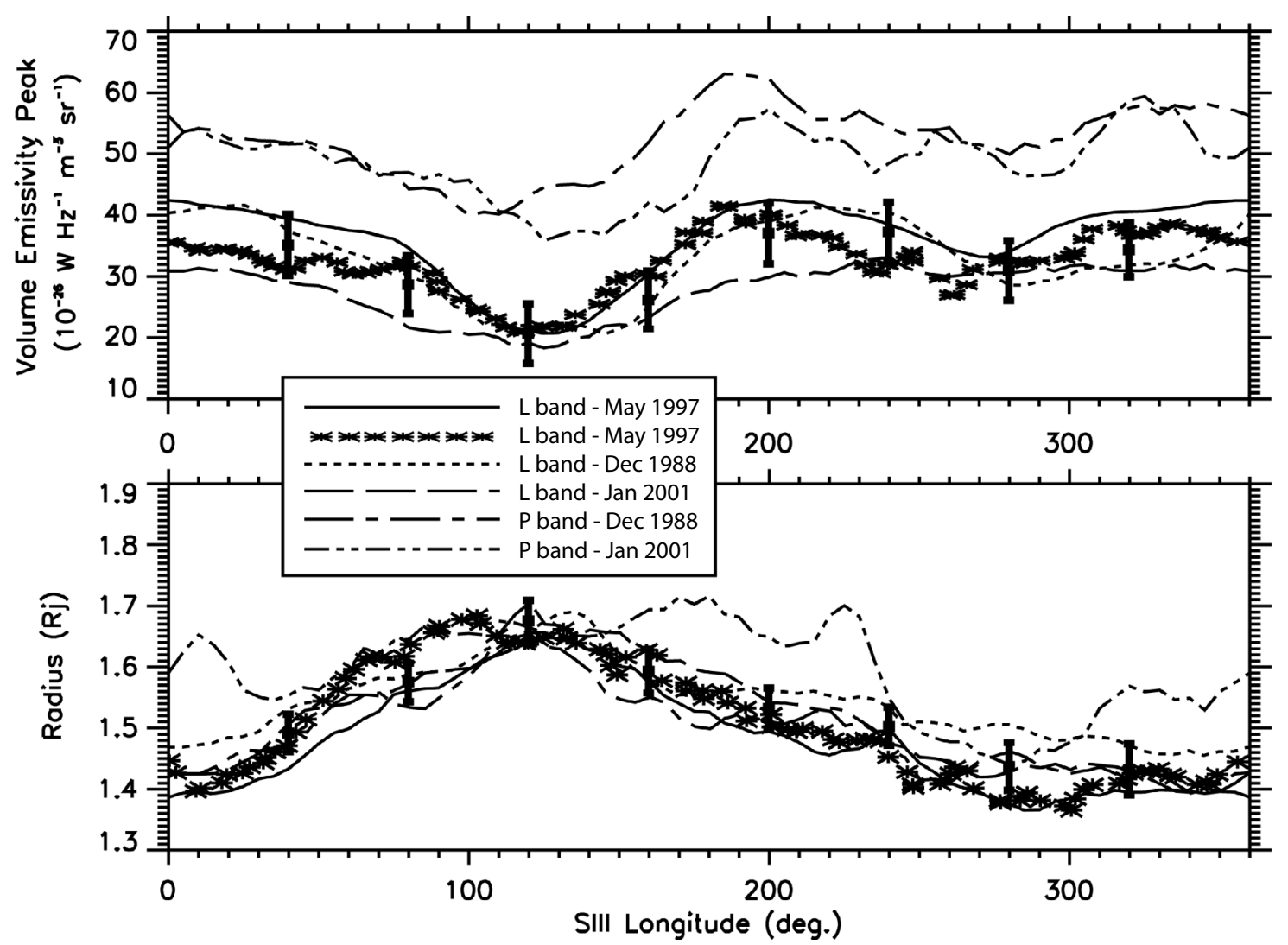

Fig. 6. Longitudinal comparisons of the brightness peak intensity (top panel) and radial position (bottom panel) of the volume emissivity maximum between radio bands and epochs. Profiles are derived from cartography of equatorial brightness distribution using either a Single Value Decomposition approach (lines) or a three-dimensional Fourier technique (star symbols) (from Figs. 5 and A.1). Error bars were calculated for the $L$-band observations and represent one standard deviation. They are placed as they would follow the mean longitudinal profiles, which are not plotted for clarity reasons.

from remote sensing and Earth-based observations and solar wind propagation codes, is underway and aims to answer the following two questions: did we witness the response of Jupiter's radiation-belt emission to an interplanetary shock (or to any other solar wind structures or solar activity) with multifrequency radio observations of Jupiter during the Cassini flyby of Jupiter? Would such a solar wind structure be capable of triggering an enhancement in the Jovian synchrotron radiation in the region $\sim 2-4 R_{\mathrm{J}}$ ? These questions are currently being addressed because preliminary data analysis results have shown that the response of the electron belt to solar radio flux is unlikely (i.e., no correlation has been found).

\section{Conclusions}

1. The 2001 VLA observations of Jupiter's synchrotron emission at $\sim 21$ and $\sim 90$ have been re-examined. The measurements from 2-3 January 2001 are compared to observations obtained on 20-21 December 1988, when viewing geometry and VLA array configurations are comparable. Comparisons show distinct differences in the features of the radio emission at both wavelengths.

2. Although differences in the brightness distribution are not observed at all longitudes, radio maps show that the Jovian synchrotron radiation was primarily less intense within the equatorial zones of maximum intensity but was spatially more extended in January 2001, resulting in higher total radiation. Radio maps indicate that changes in the spatial brightness distribution occurred during the first week of January 2001 rather than in mid-December 1988.
3. Tomographic reconstructions of equatorial brightness distributions confirm our conclusions derived from the analysis of radio maps. On 2-3 January 2001, Jupiter's synchrotron emission was different from that usually observed and variability in the spatial distribution of the electron belt was likely taking place during the first week of January 2001. Cartography also confirms an enhancement in the radiation produced by electrons magnetically trapped at distances beyond the inner ring system (beyond $\sim 2$ planetary radii) for the period 2-3 January 2001.

4. The results of our preliminary analysis of VLA data sets strongly suggest that Jupiter's synchrotron radiation was responding to external influences, such as solar wind structures, rather than to solar radio flux on a timescale of days to a couple of weeks during the Cassini flyby of Jupiter.

Acknowledgements. This research work was carried out at Southwest Research Institute (TX, USA) and was supported by the Juno mission. The authors analyzed VLA data of the NRAO observing project identification numbers AD0205, AB821, and AB0984. The VLA data are archived and made available to the public domain by the National Radio Astronomy Observatory, which is a facility of the National Science Foundation operated under cooperative agreement by Associated Universities, Inc.

\section{Appendix A: Tomographic reconstruction method validation}

In this section, we show that the Santos-Costa et al. tomographic reconstruction technique is a reliable method for mapping the equatorial brightness distribution of Jupiter's radiation-belt 

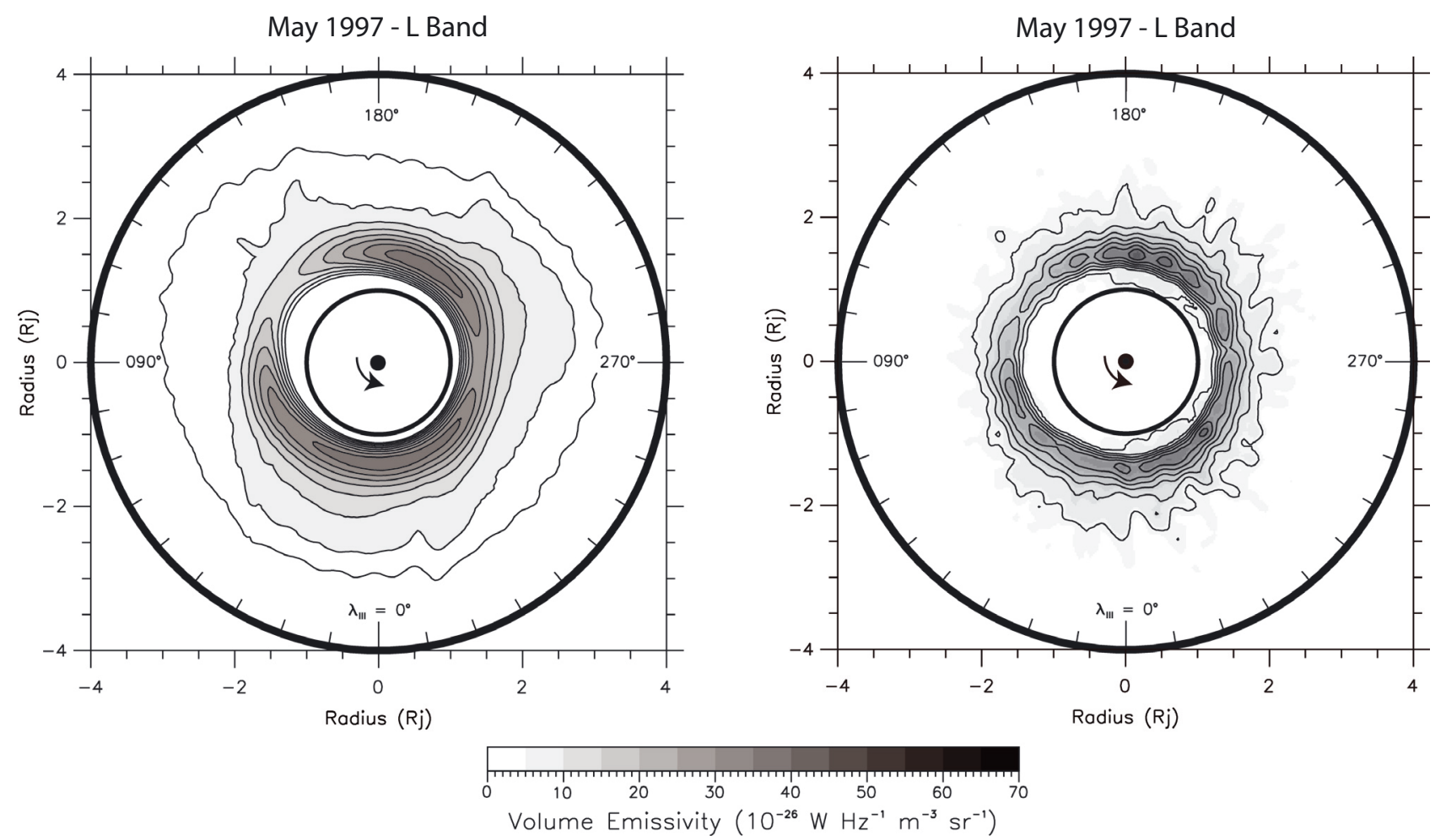

Fig. A.1. Tomographic images of the equatorial brightness distribution of Jupiter's synchrotron emission in $L$ band reconstructed for the VLA observations of May 1997. Left: using a single value decomposition approach (Santos-Costa et al. 2011b). Right: using a three-dimensional Fourier approach (Sault et al. 1997b). The north magnetic pole is indicated by the dark dot and the planet rotation with the arrow. The coordinate system is the SIII longitude. Despite some differences in the distributions (see also Fig. 6 for further comparisons), the two tomographic reconstruction techniques return compatible results, i.e., distributions projected onto the magnetic equator, and both techniques show that the equatorial radiation is primarily confined around the planet at radial distances of $\sim 1.3-1.8 R_{\mathrm{J}}$.

emission. To accomplish this, two different tomographic reconstruction techniques are applied to the VLA observations of May 1997 (e.g., Leblanc et al. 1997; Santos-Costa et al. 2009, Table 1). The results are displayed in Fig. A.1. The left-hand cartography is computed with the tomographic reconstruction technique of Santos-Costa and colleagues (Santos-Costa et al. $2011 \mathrm{~b}$ ), which is hereafter referred to as the "SVD tomo" technique. We have processed the 1997 VLA data sets as described in Sect. 2. The clean components were restored with a synthesized beam size of $7.8^{\prime \prime}$ to build every 20 degrees of CML the 18 radio maps needed to reconstruct the equatorial distribution. The right-hand cartography, which is adapted from the work of Dulk et al. (1999), was computed using a well-established threedimensional Fourier technique (Sault et al. 1997b). This second technique is hereafter referred to as the "3D Fourier tomo" technique. Both methods provide a reconstruction at a standard distance of $4.04 \mathrm{AU}$. By default, the SVD tomo technique maps the equatorial brightness distribution of the volume emissivity, which has units $\mathrm{WHz}^{-1} \mathrm{~m}^{-3} \mathrm{sr}^{-1}$, while the 3D Fourier tomo technique depicts the equatorial distribution of the brightness temperature in Kelvin units. In Figs. A.1 and 6, the conversion from brightness temperature to volume emissivity was performed using the relationship between these two physical quantities and by taking into account the grid cell size of each method. In the SVD tomo technique, the grid cell is shaped as the volume element in cylindrical coordinates and the spatial resolution is $(r \Delta \phi)(\Delta r)(\Delta z)=(\Delta \phi)(\Delta r)(\Delta z) \approx 0.35 \times 0.16 \times 0.16 R_{\mathrm{J}}$ $\left(\approx(0.21)^{3}\right)$. This grid cell size was chosen to obtain a physical solution with the SVD method and strongly depends on the number of radio maps used as inputs. Decreasing the grid cell size would result in the computation of equatorial maps biased by numerical errors without necessarily providing a finer structure (see Santos-Costa et al. 2011b, Fig. 7 and Appendix A). In the $3 \mathrm{D}$ Fourier tomo technique, the grid cells are cubic and the spatial resolution is $0.24 \times 0.24 \times 0.24 R_{\mathrm{J}}$ (Dulk et al. 1999). The $3 \mathrm{D}$ Fourier tomo technique works directly from the radio visibility data. This allows it to achieve better intrinsic resolution, but at the cost of more imaging artifacts (deconvolution errors). The finer structure in its reconstructions (particularly the fine radial features) are likely not real.

In Figs. A.1 and 5, the SVD tomo technique tends to mark off more distinctively two sectors of higher radiation that cover the System III longitudes of $\sim 180^{\circ}-240^{\circ}$ and $\sim 300^{\circ}-080^{\circ}$. To better evaluate such differences, Fig. 6 (top panel) shows a comparison between longitudinal profiles of the brightness peak intensity. These profiles show higher intensity levels at the SIII longitudes of $\sim 200^{\circ}-250^{\circ}$ and $\sim 340^{\circ}-80^{\circ}$ for the profile reconstructed with the SVD tomo technique. Nevertheless, the relative differences in intensity do not exceed $22 \%$ at these longitudes. Differences in the radial position of the maximum intensity are observed at the SIII longitudes of $\sim 40^{\circ}-110^{\circ}$, but the relative differences do not exceed $10 \%$. Despite the differences in intensity and radial position of the brightness peak at different angular sectors, profiles from the SVD tomo and 3D Fourier tomo techniques match each other within data and computational errors. Both techniques show that the equatorial radiation is primarily confined around the planet at radial distances ranging from $\sim 1.3 R_{\mathrm{J}}$ to $\sim 1.8 R_{\mathrm{J}}$. Cartography (Fig. A.1) and longitudinal profiles of the intensity and radial position of the brightness peak (Fig. 6) confirm that the two tomographic reconstruction techniques return 
compatible results and the SVD tomo technique (Santos-Costa et al. 2011b) can be used to analyze any temporal changes in the equatorial brightness distribution.

\section{References}

Berge \& Gulkis 1976, in Jupiter (Tucson: University of Arizona Press), 621 Bolton, S. J., Gulkis, S., Klein, M. J., de Pater, I., \& Thompson, T. 1989, J. Geophys. Res., 94, 121

Bolton, S. J., Janssen, M., Thorne, R., et al. 2002, Nature, 415, 987

Brecht, S. H., de Pater, I., Larson, D. J., \& Pesses, M. E. 2001, Icarus, 151, 25

de Pater 1990, in Annu. Rev. Astron. Astrophys., 28, 347

de Pater 1991, AJ, 102, 795

de Pater 2004, Planet. Space Sci., 52, 1449

de Pater, I., \& Brecht, S. H 2001, Icarus, 151, 1

de Pater, I., \& Dunn, D. E. 2003, Icarus, 163, 449

de Pater, I., \& Sault, R. J. 1998, J. Geophys. Res., 103, 19973

de Pater, I., van der Tak, F., Strom, R. G., \& Brecht, S. H. 1997a, Icarus, 129, 21

de Pater, I., Schulz, M., \& Brecht, S. H. 1997b, J. Geoph. Res., 102, 22043

Dulk, G. A., Leblanc, Y., Sault, R. J., \& Bolton, S. J. 1999, A\&A, 347, 1039

Dunn, D. E., de Pater, I., \& Sault, R. J. 2003, Icarus, 165, 121

Elachi, C., Allison, M. D., Borgarelli, L., et al. 2004, Space Sci. Rev., 115, 71

Galopeau, P. H. M., \& Gérard, E. 2001, Planet. Space Sci., 49, 1379

Gannon, J. L., Li, X., \& Temerin, M. 2005, J. Geophys. Res., 110, 12206

Gérard 1970, Radio Sci., 5, 513

Girard, J. N., Zarka, P., Tasse, C., \& Hess, S. 2012, in Proc. of the Annual meeting of the French Society of Astronomy and Astrophysics, 681

Gurnett, D. A., Kurth, W. S., Hospodarsky, G. B., et al. 2002, Nature, 415, 985

Hanlon, P. G., Dougherty, M. K., Forsyth, R. J., et al. 2004, J. Geophys. Res., $109,9 \mathrm{~S} 03$

Horne et al. 2008, Nature Phys., 4, 301

Janssen, M. A., Bolton, S. J., Levin, S. M., et al. 2001, in Proc. of the 5th International Workshop of Planetary Radio Emissions, 229
Kita, H., Misawa, H., Tsuchiya, F., Tao, C., \& Morioka, A. 2013, J. Geophys. Res., 118, 6106

Klein, M. J., Gulkis, S., \& Stelzried, C. T. 1972, ApJ., 176, L85

Klein, M. J., Thompson, T. J., \& Bolton, S. 1989, in Time-variable phenomena in the Jovian system, NASA SP, 494, 151

Klein, M. J., Bolton, S. J., Gulkis, S., et al. 2001, in Proc. of the 5th International Workshop of Planetary Radio Emissions, 221

Kloosterman, J. L., Dunn, D. E., \& de Pater, I. I. 2005, ApJS., 161, 520

Kloosterman, J. L., Butler, B., \& de Pater, I. 2008, Icarus, 193, 644

Krupp, N., Woch, J., Lagg, A., Livi, S., et al. 2004, J. Geophys. Res. 109, 9 S10 Leblanc, Y., Dulk, G. A., Sault, R. J., \& Hunstead, R. W. 1997, A\&A, 319, 274

Miyoshi, Y., Misawa, H., Morioka, A., et al. 1999, Geo. Res. Lett., 26, 9

Nichols, J. D., Bunce, E. J., Clarke, J. T., et al. 2007, J. Geophys. Res., 112, 2203

NRAO Green Book 1992, An Introduction of the NRAO Very Large Array, ed. R. M. Hjellming (NRAO Publ.)

Ott, J., \& Kern, J. 2013, CASA Synthesis \& Single Dish Reduction Reference Manual \& Cookbook, Version May 29

Pelyushenko, S. A., \& Stankevich, K. S. 1969, Sov. Astron., 13, 223

Prangé, R., Pallier, L., Hansen, K. C., et al. 2004, Nature, 432, 78

Pryor, W. R., Stewart, A. I. F., Esposito, L. W., et al. 2005, Icarus, 178, 312

Santos-Costa, D., Bolton, S. J., Thorne, R. M., Miyoshi, Y., \& Levin, S. M. 2008, J. Geophys. Res., 113, 1204

Santos-Costa, D., Bolton, S. J., \& Sault, R. J. 2009, A\&A, 508, 1001

Santos-Costa, D., Sault, R., Bolton, S., Thorne, R., \& Levin, S. 2011a, in Proc. of the 7th International Workshop of Planetary Radio Emissions, 231

Santos-Costa, D., Bolton, S. J., Sault, R. J., Thorne, R. M., \& Levin, S. M. 2011b, J. Geophys. Res., 116, 12236

Sault, R. J., Teuben, P. J., \& Wright, M. C. H. 1995, in Astronomical Data Analysis Software and Systems IV, ASP Conf. Ser., 77, 433

Sault, R. J.,Leblanc, Y., \& Dulk, G. A. 1997a, Geophys. Res. Lett., 24, 2395

Sault, R. J., Oosterloo, T., Dulk, G. A., \& Leblanc, Y. 1997b, A\&A, 324, 1190

Szego, K.,; Young, D. T., Bagdonat, T., et al. 2006, Planet. Space Sci., 54, 200

Tsuchiya, F., Misawa, H., Imai, K., \& Morioka, A 2011, J. Geo. Res., 116, 9202

Zarka 2000, in Radio Astronomy at Long Wavelengths, Geophys. Monogr. Ser., 119,167 